\title{
Spectrum of ordinary hadrons and multiquarks from QCD
}

\author{
Franco Buccella* \\ Istituto Nazionale di Fisica Nazionale, Sezione di Napoli, via Cinthia, 80126. Napoli, Italia \\ E-mail: buccelladna.infn.it
}

The description of the mass differences between the states of the baryon octet with spin parity $\left(\frac{1}{2}\right)^{+}$and decuplet $\left(\frac{3}{2}\right)^{+}$in terms of the chromomagnetic interaction is extended to the mesons $0^{-}$and $1^{-}$, to the two lowest scalar tetraquarks and to the recently discovered hidden charm pentaquarks discovered at $\mathrm{LHCb}$ with the result to predict four states $\left(\frac{3}{2}\right)^{-}$with "open channel" $\mathrm{p}$ $J / p s i$ and $\Lambda_{c} \bar{D}^{* 0}$ for the two lightest ones and $\Sigma_{c} \bar{D}^{*}$ for the two heaviest ones.

Corfu Summer Institute 2018 "School and Workshops on Elementary Particle Physics and Gravity" (CORFU2018)

31 August - 28 September, 2018

Corfu, Greece

${ }^{*}$ Speaker. 


\section{Introduction}

After the proposal that QCD is the theory of strong interactions [四], De Rujula, Georgi and Glashow [2] realized that the fine structure (the chromomagnetic interaction, CMI) accounts for the mass differences between $\Delta$ and the nucleon and between $\Sigma$ and the $\Lambda$. In this framework, there is the successful prediction :

$$
M\left(\Xi^{*}\right)-M(\Xi)=M\left(Y^{*}\right)-M(\Sigma)
$$

which had been previously obtained, by assuming the same coefficients for the mass terms transforming both as an octet for the decuplet and the octet of baryons. Applying the same approach to the charmed baryons $\Sigma_{c}$ and $\Lambda_{c}$, they predicted a mass difference high enough to allow the strong decay $\Sigma_{c}^{+} \rightarrow \Lambda_{c}+\pi^{+}$, in agreement with the discovery of both particles in a neutrino experiment [B]. The chromomagnetic interaction between the three constituent quarks gives a contribution to the mass of the baryon, they form, which depends on the quadratic Casimir of $S U(6)$ color spin, $S U(6)_{c s}, S U(3)$ color, $S U(3)_{c}$ and $S U(2)$ spin, $S U(2)_{s}$, and is proportional with a negative factor to [四] [5] :

$$
C_{6}(q q q)-\frac{C_{3}(q q q)}{2}-\frac{C_{2}(q q q)}{3}-6
$$

Since the color singlets built with three quarks with spin $\frac{1}{2}$ and $\frac{3}{2}$ belong to the 70 and 20 with Casimir $\frac{33}{4}$ and $\frac{21}{4}$, respectively, the proportionality factor is :

$$
\frac{M_{N}-M_{\Delta}}{4}
$$

The masses of $N$ and $\Delta$ may be obtained by adding the sum of the effective masses of the constituents, which is given by $\frac{M_{N}+M_{\Delta}}{2}$, since the chromagnetic contributions to their masses are opposite.

The mass difference between the $\Sigma$ and $\Lambda$ hyperons is a consequence of the different girochromomagnetic factors of the light ( $u$ and d) and strange quark (s). Indeed the total spin of the two light quarks in the $\Lambda$ is 0 and therefore the chromomagnetic contribution to the mass of that particle is equal to the one for the nucleon. Therefore the mass difference between the strange and the light constituents of the baryons is just given by the difference $M_{\Lambda}-M_{N}$. Instead for the $\Sigma$ the total spin of the two light quarks is 1 , which implies a total chromomagnetic contribution to its mass $\left[\frac{1}{6}-\frac{2 k_{s}}{3}\right]\left(M_{\Delta}-M_{N}\right)$, where $k_{s}=0.62$ is the ratio of the strange and light girochromomagnetic factors .

As long as for the charmed baryons the chromomagnetic interaction between a light and a charmed quark is weaker, mainly for the smaller gyro-chromomagnetic factor of the charmed quark inversely proportional to its mass . To reproduce the masses a factor $k_{1 c}=0.24$ is needed for the CMI and an effective mass for the charmed quark of $1715 \mathrm{MeV}$.

The $\Sigma_{b}$ and $\Lambda_{b}$ particles have a mass difference even larger, as expected.

For the mesons $\left(\pi, K \rho, K^{*}\right)$ the chromomagnetic contribution is proportional with a positive coefficient to

$$
C_{6}(q \bar{q})-\frac{C_{3}(q \bar{q})}{2}-\frac{C_{2}(q \bar{q})}{3}-4
$$


The vector and the pseudoscalar mesons belong to the 35 and to the 1 representations of $S U(6)_{c s}$ with quadratic Casimir 6 and 0, respectively, and therefore the proportionality coefficient for the mesons built with light constituents is :

$$
\frac{3\left(M_{\rho}-M_{\pi}\right)}{16}
$$

Interestingly enough, both the sign and the order of magnitude of the mass differences appearing in Eq. (ㄷ.3) and Eq. ([ए.5) have been obtained in [目] as a consequence of the sum rules proposed in [D] in the framework of the transformation, which relates constituent and current quarks [8] [Q] . The chromomagnetic contribution for the strange pseudoscalar is proportional to $k_{s}$ and one expects

$$
\frac{M_{K^{*}}-M_{K}}{M_{\rho}-M_{\pi}}=k_{s}
$$

The sum of the light constituent masses in the mesons is given by $\frac{3 M_{\rho}+M_{\pi}}{4}$, while for the strange mesons is given by $\frac{3 M_{K^{*}}+M_{K}}{4}$. So one needs a larger chromomagnetic interaction and a smaller effective masses for the light and the strange quarks than in the case of the baryons. Both these properties can be understood by the more intense chromoelectric attraction between a quark and an antiquark, which form a color singlet with respect to two quarks, which combine in a $\overline{3}$ of $\mathrm{SU}(3)$ color. Indeed, the stronger attraction implies a smaller constituent mass and a larger contact interaction. In fact, for the charmed mesons $D$ and $D^{*}$, a slightly smaller mass, $1615 \mathrm{MeV}$, and larger $k_{2 c}=0.26$ are needed with respect to the charmed baryons. Also, the values found for the $c \bar{c}$ states, $1535 \mathrm{MeV}$ for the mass of the charmed quark and $K_{c}^{2}=0.186$ for the square of the gyrochromomagnetic factor can be understood as a consequence of the smaller distance between the constituents.

The mass of $3621.40 \mathrm{MeV}$ of the $\Xi_{c c}^{++}$recently found by $L H C_{b}$ [ए]] implies an effective mass of the constituent charmed quarks of $1665 \mathrm{MeV}$, somewhat smaller than the one found for the charmed mesons and $\Lambda_{c}$.

For the two nonets of scalar tetraquarks, where the isoscalar states built with the light constituents are the $f^{0}(600)$ and $f^{0}(1370)$ resonances, their masses are reproduced with an effective chromomagnetic interaction as for the baryons and with a larger constituent mass. Interestingly enough, this explains why the lowest one, which decays into two pions, has a very large width, while the other one decays mainly into four pions [W]]. In fact, the $S U(6)_{c s}$ Casimir, which gives the most important chromomagnetic contribution to the masses, implies that the lighter state is almost a $S U(6)_{c s}$ singlet with an "open channel" [12] into two pions, which are also $S U(6)_{c s}$ singlets, while the heavier one transforms mainly as a 405 and therefore has an open channel into a pair of $\rho$

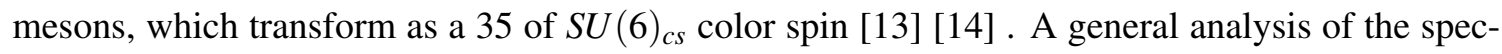
trum of negative and positive pentaquarks built with the three lightest quarks can be found in [प5]] and the study of $3 q 3 \bar{q}$ hexaquarks in [ए]]. We may be confident that also the specrum of the hidden charm pentaquark states is well described by the chromo-magnetic interaction.

The dependance of both the constituent masses and of the normalization of the CMI contributions to the mass splitting on the number of constituents in s-wave will be kept in account, when we shall study their spectrum . In the next section we shall describe the formation of the hidden charm pentaquarks discovered at $\mathrm{LHCb}$. In the third section, we shall compute the masses of the four 
$\left(\frac{3}{2}\right)^{-}$hidden charm pentaquarks and the "open channels" for their decays. Our description will account for their different widths. In the fourth section, we shall give reasons why only some of the multiquark states have been detected. Then, we shall give our conclusions.

\section{The pentaquarks discovered at $L H C_{b}$}

The discovery of two pentaquarks with hidden charm in the decay [ㅍ] :

$$
\Lambda_{b} \rightarrow p+J / \psi+K^{-}
$$

confirms the propensity of particles with beauty to give rise to multi-quark states with hidden charm, as previously seen [ए8] for the $\left(3872,1^{+}\right)$resonance decaying into $J / \psi+\rho^{0}$ (or $\omega$ ) along with a kaon in the decay of $B_{q}$ 's. The study of states with non-minimal number of constituents

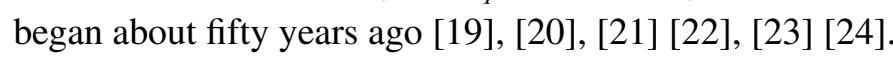

The existence of the $\Theta^{+}, Y=2$ baryon resonance has been confirmed [25] and the large crosssection at high momentum transfer for the production of $\left(3872,1^{+}\right)$resonance [26] shows that it is a compact object [27] and not a molecule, that should behave as the deuteron, and, as such, should be very rarely produced at high momenta [28] . This seems to confirm the $[2 q 2 \bar{q}]$ configuration for the $\left(3872,1^{+}\right)$, as described in [29] and [14]]).

The production of hidden charm multiquarks is associated with the Cabibbo allowed process for the decaying $\Lambda_{b}$ with an amplitude proportional to $V_{c b}^{*} V_{c s}$ :

$$
b \rightarrow c+s+\bar{c}
$$

followed by the emission of a gluon by the strange quarks, giving rise to a $q \bar{q}$ pair for the decay of $\bar{B}^{0}$ into $K_{S}^{0}+3872,1^{+}$[B]] or to a $u \bar{u}$ pair for the decay into a $K^{-}+$the hidden charm pentaquarks $4380,\left(\frac{3}{2}\right)^{-}$and $4450,\left(\frac{5}{2}\right)^{+}$discovered at $L H C b$ [B] $]$. The $1^{+}$resonance is built by the merging of the two $c \bar{c}$ and $q \bar{q}$ color octets of spin 1, while the pentaquarks by the union of the $c \bar{c}$ octet with the color and flavor octet with spin parity $\left(\frac{1}{2}\right)^{+}$built by the spectator diquark in the $\Lambda_{b}$ and the $\mathrm{u}$, produced by the gluon, while the $\bar{u}$ forms with the strange quark produced in the decay a $K^{-}$.

If the five constituents join in relative S-wave, they may give rise to $\frac{3}{2}^{-}$hidden charm pentaquarks, while if the two octets are in a P-wave they may give rise to the $\frac{5}{2}^{+}$[B] . In the first case, when they join, they give rise to a combination of eigenstates of the QCD hamiltonian. In both cases isospin conservation requires that the three light quarks have $I=\frac{1}{2}$. Therefore, the Pauli principle demands that, if they are in S-wave with a symmetric wave function, they transform as the 70 representation of $S U(6)_{c s}$ (for three objects, a mixed symmetry state may give rise to an antisymmetric one only by multiplying it by another mixed symmetry state) [团] [可] [32] .

The $c \bar{c}$ pair transforms as the $35+1$ representation of $S U(6)_{c s}$.

The mechanism for the decay of the $\left(\frac{5}{2}\right)^{+}$pentaquark is similar to the decay of the $1^{+}$tetraquark at 3872 into $J / \psi+\rho^{0}$ (or $\omega$ ) : a gluon exchange, which turns the two color octets into singlets.

Instead, their formation in the decay of $B_{q}$ and $\Lambda_{b}$ is different. In fact, in the first case the strange quark produced in the $b$ decay, forms a kaon together with the spectator antiquark, while the $q \bar{q}$ pair produced by the gluon forms together with the $c \bar{c}$ the $\left(3872,1^{+}\right)$tetraquark. The analogous process for the $\Lambda_{b}$ decay, with the strange quark forming with the spectator diquark a $\Lambda$, would 
give rise to the decay $\Lambda_{b} \rightarrow \Lambda+\left(3872,1^{+}\right)$[3] ] .

Högaasen and Sorba [四] studied all the possibilities with three constituents in P-wave with respect to the other two and came to the conclusion that the most interesting case is with two color octets of the three light quarks and the $c \bar{c}$ pair, with the caveat that each octet might be turned into an ordinary hadron by absorbing a gluon before combining to form the hidden charm pentaquark. One should keep in mind, however, the fact that, in the decay

$$
\Lambda_{b} \rightarrow p+J / \psi+K^{-}
$$

a gluon should be emitted and turned into a $(u \bar{u})$ pair to give rise to the seven final constituents and therefore the presence of another gluon requires a higher order in QCD.

The seven constituents may also combine in a different way with the strange quark giving rise to a $\Lambda$ and the $c \bar{c}$ and the $u \bar{u}$ color octets to the $\left(3872,1^{+}\right)$. The decay

$$
\Lambda_{b} \rightarrow \Lambda+\left(3872,1^{+}\right)
$$

might be looked for in final states $p+\pi^{-}(\Lambda), \mu^{+} \mu^{-}(J / \psi)$ and $\pi^{+} \pi^{-}\left(\rho^{0}\right)$ [B]] .

As we shall stress in the following sections, the "beautiful" particles. due to their relative long lifetime, decay at a distance from the interaction point sufficient enough to avoid the presence of the gluons emitted there.

\section{Formation, masses and decays of the hidden charm pentaquarks}

The Cabibbo allowed process for the decaying $\Lambda_{b}$ described in Eq. (ㅁ. $($ ) is induced by the term of the non-leptonic lagrangian proportional to :

$$
\overline{c_{L}} \gamma_{\mu} b_{L} \overline{s_{L}} \gamma^{\mu} c_{L}
$$

which is a combination of

$$
\overline{c_{L}} \gamma_{\mu} c_{L} \overline{s_{L}} \gamma^{\mu} b_{L}
$$

and

$$
\overline{c_{L}} \gamma_{\mu} \lambda_{a} c_{L} \overline{S_{L}} \gamma^{\mu} \lambda_{a} b_{L}
$$

as a consequence of the $S U(3)$ crossing relations. The term in Eq. (B.2) gives rise to the amplitude for the "golden channel" for CP violation $(\bar{B})^{0} \rightarrow J / \psi+K_{S}^{0}$, the term in Eq. (B.3) is the first step for the decay $\Lambda_{b} \rightarrow J / \psi+p+K^{-}$. In fact the emission by the strange quark of a gluon converted into a $u \bar{u}$ pair completes the number of constituents, seven, needed to hadronize into the final particles. The scale, at which the gluon is emitted and converted needs not to be high and therefore the factor $\alpha_{s}$ needs not to be too small. The produced $c$ quark, if it does not recombine with the spectator diquark $u d$ to give a $\Lambda_{c}$, may form a color octet with spin 1 with the $\bar{c}$ [B]]. The $\bar{u}$ produced by the gluon may combine with the $s$-quark to form the negative kaon, while the $u$ with the spectator diquark in the $\Lambda_{b}$ may form a color octet with spin $\frac{1}{2}$ and the same flavor of the proton. The two color octets may give rise to one or the other of the two resonances, depending on their relative orbital momentum, the one with negative parity for the S-wave, the one with positive parity for the 
P-wave. With all the constituents in $\mathrm{S}$-wave, one has four states with $S=\frac{3}{2}$, which can be obtained by the products $\frac{3}{2} \times 1, \frac{3}{2} \times 0$ and $\frac{1}{2} \times 1$. Let us remember that the 70 contains both $\frac{3}{2}$ and $\frac{1}{2}$ spin color octets and a spin $\frac{1}{2}$ singlet, while the 35 contains both 1 and 0 spin color octets and a spin 1 color singlet. So we have the following possibilities for the color-spin transformation properties of the three light quarks and the $c \bar{c}$ pair :

$$
\begin{aligned}
& \left(8, \frac{3}{2}\right) \times(8,1) \text { combined into a }\left(1, \frac{3}{2}\right) \\
& \left(8, \frac{3}{2}\right) \times(8,0) \text { combined into a }\left(1, \frac{3}{2}\right) \\
& \left(8, \frac{1}{2}\right) \times(8,1) \text { combined into a }\left(1, \frac{3}{2}\right) \\
& \left(1, \frac{1}{2}\right) \times(1,1) \text { combined into a }\left(1, \frac{3}{2}\right)
\end{aligned}
$$

The total contribution of CMI is given by [B] :

$$
M=M(70)+M(70 \times 6)+M(70 \times \overline{6})+M(6 \times \overline{6})
$$

The four terms give the contribution of the chromomagnetic interaction of the three light quarks, of their interaction with the $c$ quark and the $\bar{c}$ antiquark and of the interaction of the two heavy constituents, $c$ and $\bar{c}$, respectively. The first and the fourth terms of Eq. (3.8) are given by :

$$
M(70)=-\frac{m_{\Delta}-m_{N}}{4}\left[C(70)_{6}-\frac{C(3 q)_{3}}{2}-\frac{C(3 q)_{2}}{3}-6\right]
$$

and

$$
M(6 \times \overline{6})=\frac{3}{16}\left(M_{J / \psi}-M \eta_{c}\right)\left[C(35)_{6}-\frac{C(c \bar{c})_{3}}{2}-\frac{C(c \bar{c})_{2}}{3}-4\right]
$$

To consider the chromomagnetic interaction between the charmed and the light quarks one has to keep account of their different chromo-magnetic factors [33] [5] . The sum of the contributions of the first and the fourth terms to the states defined in Eq. (B.4) ...Eq. (B..7) is given by :

$$
M(70)+M(6 \times \overline{6})=\frac{m_{\Delta}-m_{N}}{8}-\frac{m_{J / \psi}-m_{\eta_{c}}}{32}
$$

for the eigenstate defined in Eq. (B.4)

$$
M(70)+M(6 \times \overline{6})=\frac{m_{\Delta}-m_{N}}{8}+\frac{3\left(m_{J / \psi}-m_{\eta_{c}}\right)}{32}
$$

for the eigenstate defined in Eq. (B.5)

$$
M(70)+M(6 \times \overline{6})=-\frac{M_{\Delta}-M_{N}}{4}-\frac{M_{J / \psi}-M_{\eta_{c}}}{32}
$$

for the eigenstate defined in Eq. (B.6), while for the "open channel" eigenstate defined in Eq. (B.]) the sum is :

$$
M(70)+M(6 \times \overline{6})=\frac{M_{N}-M_{\Delta}}{2}+\frac{M_{J / \psi}-M_{\eta_{c}}}{4}
$$


The second and the third term in Eq. (B.8) are related to the chromo-magnetic interaction of the light quarks with $c$ and $\bar{c}$, and are proportional to $k_{1}=0.24$ and $k_{2}=0.26$, the values of $k_{c}$ for charmed baryons and mesons, respectively. To evaluate them one should consider the tensor products :

$$
\begin{gathered}
70 \times 6=210+105+105^{\prime} \\
70 \times \overline{6}=384+21+15
\end{gathered}
$$

and the fact that

i) the $(3,5)$ of $S U(6)_{c s}$ is contained in the $105^{\prime}$,

ii) the three $(3,3)$ in the three representation of the first product,

iii) one of the $(\overline{3}, 3)$ is contained in the 15 ,

iv) the $(\overline{3}, 5)$ and the other two $(\overline{3}, 3)$ 's in the 384 for the second product.

Therefore the contributions of the second term is given by :

$$
\frac{k_{1}\left(M_{N}-M_{\Delta}\right)}{4}\left[C(3 q c)_{6}-\frac{C(3)_{3}}{2}-\frac{C(3 q c)_{2}}{3}-C(70)_{6}+\frac{C(3 q)_{3}}{2}+\frac{C(3 q)_{2}}{3}-2\right]
$$

while the contributions of the third term is given by :

$$
\frac{3 k_{2}\left(M_{\rho}-M_{\pi}\right)}{16}\left[C(3 q \bar{c})_{6}-\frac{C(\overline{3})_{3}}{2}-\frac{C(3 q \bar{c})_{2}}{3}-C(70)_{6}+\frac{C(3 q)_{3}}{2}+\frac{C(3 q)_{2}}{3}-2\right]
$$

The quadratic Casimir of the $S U(6)$ representations 15, 21, 105', 105, 210 and 384 are $\frac{14}{3}, \frac{20}{3}$, $\frac{26}{3}, \frac{32}{3}, \frac{38}{3}$ and $\frac{35}{3}$, respectively, while the relevant CG for the tensor products are reported in the Appendix of [B]].

In conclusion, the terms proportional to $\left(M_{\Delta}-M_{N}\right)$ and to $\left(M_{\rho}-M_{\pi}\right)=\frac{1}{K_{c}^{2}}\left(M_{J / \psi}-M_{\eta_{c}}\right)$ are the matrices respectively shown in Tables $\square$ and 凹, while in Table 3$]$ we show the numerical evaluation of Eq. (B.8) in $\mathrm{MeV}$ in the base of the states .

$$
\begin{aligned}
& |1>=| 70 \times 6,(8,4) \times(3,2) \rightarrow(3,5)> \\
& |2>=| 70 \times 6,(8,4) \times(3,2) \rightarrow(3,3)> \\
& |3>=| 70 \times 6,(8,2) \times(3,2) \rightarrow(3,3)> \\
& |4>=| 70 \times 6,(1,2) \times(3,2) \rightarrow(3,3)>
\end{aligned}
$$

with eigenvalues : - 120, - 71, 11 and $80 \mathrm{MeV}$ in correspondence to the eigenvectors [B] :

$(.057, .08, .59, .624)$

$(.225, .063, .604,-.762)$ 


\begin{tabular}{|c|c|c|c|}
\hline$\frac{1+3 k_{1}}{8}$ & 0 & 0 & 0 \\
\hline 0 & $\frac{1-3 k_{1}}{8}$ & $\frac{k_{1}}{3}$ & $\frac{k_{1}}{6}$ \\
\hline 0 & $\frac{k_{1}}{3}$ & $-\frac{1+3 k_{1}}{8}$ & $\frac{k_{1}}{6}$ \\
\hline 0 & $\frac{k_{1}}{6}$ & $\frac{k_{1}}{6}$ & $\frac{k_{1}-1}{2}$ \\
\hline
\end{tabular}

Table 1: CMI contributions proportional to $\left(M_{\Delta}-M_{N}\right)$

\begin{tabular}{|c|c|c|c|}
\hline$\frac{3\left(-9 k_{2}+K_{c}^{2}\right.}{64}$ & $-\frac{\sqrt{15}\left(3 k_{2}+K_{c}^{2}\right)}{64}$ & $-\frac{\sqrt{15} k_{2}}{8}$ & $-\frac{\sqrt{15} k_{2}}{16}$ \\
\hline$-\frac{\sqrt{15}\left(3 k_{2}+K_{c}^{2}\right)}{64}$ & $-\frac{\left(15 k_{2}+K_{c}^{2}\right.}{64}$ & $-\frac{\sqrt{15} k_{2}}{8}$ & $-\frac{\sqrt{15} k_{2}}{16}$ \\
\hline$-\frac{\sqrt{15} k_{2}}{8}$ & $-\frac{k_{2}}{8}$ & $-\frac{\left(3 k_{2}-K_{c}^{2}\right.}{32}$ & $-\frac{k_{2}}{8}$ \\
\hline$-\frac{\sqrt{15} k_{2}}{16}$ & $-\frac{k_{2}}{16}$ & $-\frac{k_{2}}{8}$ & $\frac{K_{c}^{2}}{4}$ \\
\hline
\end{tabular}

Table 2: CMI contributions proportional to $\left(M_{\rho}-M_{\pi}\right)$

\begin{tabular}{|c|c|c|c|}
\hline 10.5 & -33 & -71.5 & -35.05 \\
\hline-33 & 28.75 & 5.3 & 2.65 \\
\hline-71.1 & 5.3 & -53.2 & 6.5 \\
\hline-35.05 & 2.65 & 6.5 & -86.2 \\
\hline
\end{tabular}

Table 3: Numerical evaluation of Eq.(12)

$(.39, .847,-.35,-.094)$

$(.736,-.522,-. .041,-.15)$

The "open channel" $p+J / \psi$ has negligible components along the two eigenvectors corresponding to the two higher eigenvalues and substantial ones along the two lower ones. This agrees well with the mass of the $\left(\frac{3}{2}\right)^{-}$state if we take the constituent masses of the light quarks from the lowest baryons, and those of $c$ and $\bar{c}$ from the $\Lambda_{c}$ and from the lowest charmed mesons. Namely, extracting the sum of the constituents masses from :

$$
\frac{M_{\Delta}-M_{N}}{2}+M_{\Lambda_{c}}+\frac{3 M_{D^{*}}+M_{D}}{4}=4480 M e V
$$

implies for the two lightest $\left(\frac{3}{2}\right)^{-}$states a mass of 4360 and $4409 \mathrm{MeV}$.

It can be noticed that by taking the masses of charmed constituents from charmonium would lead to smaller constituent masses, but the presence of the three light quarks favors us to consider charmed baryon and mesons. The tendency of larger constituent masses with the increasing number of constituents in relative $\mathrm{S}$-wave may give rise to a larger global constituent mass.

The value found here has an important consequence, as it predicts two higher $\left(\frac{3}{2}\right)^{-}$states at 4491 and $4560 \mathrm{MeV}$.

By considering $q q c-\bar{c} q$ combinations, the "open channels" $\left(\Lambda_{c} D^{\bar{*} 0}\right)$ and the $I=\frac{1}{2}$ combination $\frac{1}{\sqrt{3}}\left(\sqrt{2} \Sigma_{c}^{++} D^{\bar{*}-}-\Sigma_{c}^{+} \bar{D}^{-0}\right)$ have different components along the CMI eigenvectors. While $\Lambda_{c} \bar{D}^{* 0}$ with total spin $\frac{1}{2}$ for the light quarks is a combination of the two last vectors and therefore has substantial components along the two lower mass eigenstates, $\frac{1}{\sqrt{3}}\left(\sqrt{2} \Sigma_{c}^{++} D^{\bar{*}}\right)-\Sigma_{c}^{+} D^{\bar{*} 0}$ has com- 
ponents mainly along the two states with spin $S(u u d)=\frac{3}{2}$ for the light quarks, as can be seen from the identity for the states with $S=\frac{3}{2}$ [B] :

$$
\begin{aligned}
\mid S(\text { иu })=1, S(\text { иис })=\frac{1}{2}, S(c \bar{c})=1>= & \frac{1}{3}\left[\sqrt{5} \mid S(\text { uиd })=\frac{3}{2},|S(c \bar{c})=1>-\sqrt{3}| S(\text { uиd })=\frac{3}{2} S(c \bar{c})=0>\right. \\
& \left.+\mid S(\text { uиd })=\frac{1}{2}, S(c \bar{c})=1>\right]
\end{aligned}
$$

The relationship between the $(8 \times 8)_{1}$ and $1 \times 1$ for $(u u d) c \bar{c}$ and $(u u c) d \bar{c}$ is supplied by the well known $S U(3)$ identities :

$$
\begin{aligned}
\delta_{\alpha}^{\beta} \delta_{\gamma}^{\varepsilon} & =\frac{1}{3} \delta_{\alpha}^{\varepsilon} \delta_{\gamma}^{\beta}+\frac{1}{2}\left(\lambda_{a}\right)_{\alpha}^{\varepsilon}\left(\lambda_{a}\right)_{\gamma}^{\beta} \\
\left(\lambda_{a}\right)_{\alpha}^{\beta}\left(\lambda_{a}\right)_{\gamma}^{\varepsilon} & =\frac{16}{9} \delta_{\alpha}^{\varepsilon} \delta_{\gamma}^{\beta}-\frac{1}{3}\left(\lambda_{a}\right)_{\alpha}^{\varepsilon}\left(\lambda_{a}\right)_{\gamma}^{\beta}
\end{aligned}
$$

The fact that the chromomagnetic interaction for the light quarks (the ones with the higher gyrochromomagnetic factor) gives a positive contribution to the state $\Sigma_{c} \bar{D}^{*}$ and negative for $\Lambda_{c} \bar{D}^{0 *}$ (in analogy with the large difference $M_{\Sigma_{c}}-M_{\Lambda_{c}}$ [] [ []] ) leads us to guess that the $\Sigma_{c} \bar{D}^{*}$ and $\Lambda_{c} \bar{D}^{0 *}$ "open channels" have large components along the 4560 and $4360 \mathrm{MeV}$ resonances, respectively. However, according to the formation mechanism starting from the third state $\left(8, \frac{1}{2}\right) \times(8,1)$, which has a negligible component along the higher eigenstate, the $\Sigma_{c} \bar{D}^{*}$ decay may be more easily seen for the 4491 resonance. For the decay of the $\Sigma_{c}^{++}$we may have the same sequence, namely :

$$
\begin{gathered}
\Sigma_{c}^{++} \rightarrow \Lambda_{c}+\pi^{+}, \\
\Lambda_{c} \rightarrow p+K^{-}+\pi^{+}
\end{gathered}
$$

which has led to the discovery of $\Sigma_{c}^{++}$in a neutrino experiment [B] .

The narrow width of the $4450, \frac{5}{2}^{+}$can be explained by the fact that the decay into $p+J / \psi$ needs the exchange of one gluon, as it happens for the decay of the $(3872), 1^{+}$) into $J / \psi+\rho^{0}$ (or $\omega$ ), if one identifies it as the state built with the light $(q \bar{q})$ and the charmed $(c \bar{c})$ pairs transforming as the $(8,3)$ representation of $S U(3)_{c} \times S U(2)_{s}$ [29].

The mechanism of formation of the strange isoscalar pentaquark with hidden charm is similar to the description of the formation of the $38721^{+}$in B decays [B0], with the strange quark produced in the weak decay forming a strange color octet together with the scalar and iso-scalar spectator in $\Lambda_{b}$.

Notice that, in general, it is not easy to produce hadrons with non minimal number of constituents, since the $q$ and $\bar{q}$ produced by the gluons tend to combine very fast into color singlets and the easiest way is to form ordinary hadrons. In Cabibbo allowed B decays, the creation of a $c \bar{c}$ color octet pair, which exerts an attraction on another octet built with a $q \bar{q}$ pair or three light quarks, can give rise to hadron states with hidden charm.

In conclusion, the interpretation of the two pentaquark resonances with hidden charm, discovered at $L H C b$ [प]], as built with a $c \bar{c}$ and three light quark in S-wave for the $\left(\frac{3}{2}\right)^{-}$, and with the five constituents in P-wave for the $\left(\frac{5}{2}\right)^{+}$, accounts for their different widths. An important consequence of this description is the prediction of two $\left(\frac{3}{2}\right)^{-}$resonances at a mass of 4360 and $4560 \mathrm{MeV}$, with large components along the "open channels" $\Lambda_{c} \bar{D}^{+0}$ and $\Sigma_{c} \bar{D}^{*}$ final states, respectively. 
As we shall stress in the following section, due to their relative long lifetimes the "beautiful" particles decay at a distance from the interaction point sufficient enough to avoid the presence of the gluons emitted there. Such gluons give rise to $q \bar{q}$ pairs transforming as color octets with the $q$ 's and the $\bar{q}$ 's, which build ordinary hadrons with the other constituents.

\section{Formation of multiquark states}

The fact that the $\left(3872,1^{+}\right)$, which is a compact object since it is produced also at high $p_{T}$, unlike the deuteron, and is seen only through its neutral component, shows the relevance of the formation of multiquark states. In fact, the mechanism described in the previous sections is operative only for the neutral component [30], which is indeed the only component discovered.

As for the states predicted by Jaffe [12], strong evidence concerns the two scalar tetraquark nonets, namely:

(i) the multiplet consisting of $f^{0}(600), \kappa(770)$ and $f^{\prime 0}$ and $a^{0}$, degenerate as expected, at $980 \mathrm{MeV}$ (ii) another one for which $f^{0}(1370)$ is the isoscalar resonance consisting of light constituents.

As discussed before, our interpretation is that this second scalar nonet transforms almost like a 405 of $S U(6)_{c s}$, and thus it has an "open channel" into two $\rho$ 's, which transform as a $35(35 \times 35=$ $1+\ldots 405)$. Albeit being below threshold, the large $\rho$ width makes the $f^{0}(1370)$ appear in 4 pions, as follows from the analysis in [ए]].

The fact that tetraquarks with light constituents and higher spin have not yet been seen led the Rome group [34] to consider only the diquarks transforming as $(\overline{3}, S=0, \overline{3})$ with respect to $S U(3)_{c} \times$ $S U(2)_{s} \times S U(3)_{f}$ and their antiparticles, which may give rise to only one scalar nonet. To account for the heavier one, they advocate an instanton [B5]. As we have shown in the second section, the masses and decays of the two states built with the light constituents are well described by deducing their spectrum with the same approach followed in [■] for ordinary hadrons. In fact, when the $\overline{3}, S=0$ and $3, S=0$ join, they give rise to a superposition of eigenstates of the CMI, with "open channel" [ए2] two pions or two $\rho$ 's, respectively.

To build the $\left(3872,1^{+}\right)$, the Rome group considered also diquarks transforming as a $(\overline{3}, 3)$ under $S U(3)_{c} x S U(2)_{s}$ [36]. For these diquarks the chromo-electric force is attractive, while the chromomagnetic is repulsive, which makes their formation less probable. Moreover, as well as the diquark $(\overline{3}, 1)$, they may combine with a quark to form a baryon.

The mechanism proposed here for the formation of the $\left(3872,1^{+}\right)$, which accounts for the fact that only its neutral component has been found, is according to us better motivated. The tendency of the diquark $(\overline{3}, 3)$ to form a baryon with the quark, rather than combine with a $(\overline{3}, 1)$ diquark to build a spin 1 state or with a $(\overline{3}, 3)$ diquark to give rise to spin and (or) isospin 2 states explains why the large class of states predicted by Jaffe has not yet been found.

According to us, the approach introduced in [] for ordinary baryons can be successfully extended to find the spectrum of multiquark states . However, a production mechanism is needed to prevent their formation from being overwhelmed by the recombination of the $\mathrm{q}$ and $\bar{q}$ produced by the gluons into ordinary hadrons. For this reason, the decays of particles with beauty, produced at Belle and $\mathrm{BaBaR}$, and also at $L H C b$, offer a favourable opportunity, since the "beautiful" particles decay in absence of associated production. This is evident for the $e^{+} e^{-}$rings, but it happens also for the particles produced at $L H C b$, since the long lifetime of the b quark allows the hadrons with 
beauty to leave the interaction point before decaying, with the consequence that the products of their decays are not surrounded by the $q \bar{q}$ pairs produced in the interaction.

\section{Conclusions.}

The analysis presented in this paper leads us to the following conclusions:

(i) The approach based on the chromo-magnetic interaction to find the spectrum of the multiquark states, applied successfully to the lowest scalar nonets [[4] and to the $\left(3872,1^{+}\right)$[ख2] implies the existence of four $\left(\frac{3}{2}\right)^{-}$hidden charm pentaquarks with "open channels" $\mathrm{p} J / \psi$ and $\Lambda_{c}$ $\bar{D}^{* 0}$ for the two lighter ones and $\Sigma_{c} \bar{D}^{*}$ for the two heavier ones .

(ii) The smaller width of the $\left(\frac{5}{2}\right)^{+}$is explained by the gluon exchange needed for its decay.

(iii) The property of the beautiful particles of traveling away from the interaction point, as a consequence of their lifetime, prevents the formation of hidden charm multiquarks through their Cabibbo favored decay with the production of a pair $c \bar{c}$ and a strange quark from being overwhelmed by the production of ordinary hadrons.

\section{Acknowledgement.}

I am very grateful to Prof. Mario Abud for his explanation of the non detection of the charged partners of the 3872, which inspired the considerations on the relevance of the formation mechanism of multiquark states.

\section{References}

[1] H. Fritzsch, M. Gell-Mann and H. Leutwyler, Phys. Lett. B47 (1973) 365 .

[2] A. De Rújula, H. Georgi and S.L. Glashow, Phys. Rev. D12 (1975) 147.

[3] E. G. Cazzoli, A. M. Cnops, P. L. Connolly, R. I. Louttit, M. S. Murtagh, P. A Palmer, N. P. Samios, T. T. Tao and H. H. Williams, Phys. Rev. Lett. 34, (1975) 1125.

[4] H. Högasen and P. Sorba, Nucl. Phys. B145 (1978) 119 .

[5] M. de Combrugghes, H. Högaasen and P. Sorba, Nucl. Phys. B156 (1979) 347.

[6] F. Buccella, F. Nicolo' and A. Pugliese Lettere al Nuovo Cimento. 8 (1973) 244.

[7] S. Weinberg, Phys. Rev. 177 (1968) 2604.

[8] F. Buccella, E. Celeghini, H. Kleinert, C. A. Savoy and E. Sorace Nuovo Cimento A69 (1970) 133.

[9] F. Buccella, E. Celeghini and C. A. Savoy Nuovo Cimento A70 (1972) 281.

[10] R. Aaij et al.(LHCb Collaboration), it Phys. Rev. Lett. 119 (2017) 112001 .

[11] M. Gaspero, Nucl. Phys. A562 (1975) 407.

[12] R. L. Jaffe, Phys. Rev. D17 (1978) 1444.

[13] F. Buccella, Mod. Phys. Lett. A21 (2006) 831. 
[14] F. Buccella, H. Högaasen, J. M. Richard and P. Sorba Eur. Phys. J. C49 (2007) 743.

[15] M. Abud, F. Buccella, D. Falcone, G. Ricciardi and F. Tramontano, Adv. Stud. Theor. Phys. 2 (2008) 929.

[16] M. Abud, F. Buccella and F. Tramontano, Phys. Rev. D81 (2010) 0744018 .

[17] R. Aaij et al.(LHCb Collaboration), it Phys. Rev. Lett. 115 (2015) 072001 .

[18] S. K. Choi et al. (Belle Collaboration), Phys. Rev. Lett. 91 (2003) 252001.

D. Acosta et al. (CDF Collaboration), Phys. Rev. Lett. 93 (2004) 072001.

V. M. Abazov et al. (D0 Collaboration), Phys. Rev. Lett. 93 (2004) 162002.

B. Aubert et al. (BaBar Collaboration), Phys. Rev. D71 (2005) 071103.

R. A. Aaij al. (LHCb Collaboration), Phys. Rev. Lett. 110 (2013) 222001.

[19] J. Rosner Phys. Rev. Lett. 21 (1968) 950 .

[20] R. L. Jaffe, Phys. Rev. D15 (1977) 267 and 281 .

[21] H. M. Chan and H. Högaasen Phys. Lett. bf B76 (1978) 634 .

[22] R. L. Jaffe, Phys. Rev. Lett. bf 38 (1977) 195 and 617

[23] V. A. Matveev and P. Sorba, Lett.Nuovo Cimento 20121 (1977) \& Nuovo Cimento A45 (1978) 257.

[24] H. Högaasen and P. Sorba, Fizika B14 (2005) 2245.

[25] Ya. I. Azimov, J. Phys. G37 (2010) 023001 and Proceedings of the International Workshop "Hadron Structure and QCD" 158 edited by V. T. Kim and L. V. Lipatov.

[26] S. Chatchyan et al. (CMS Collaboration), J. High Energy Phys. D4 (2013) 154.

[27] A. Esposito, A.L. Guerrieri, L. Maiani, F. Piccinini, A. Pilloni, A. D. Polosa and V. Riquer, Phys. Rev. D92 (2015) 034028 .

[28] J. Adam et al. (ALICE Collaboration), arXiv : 150608453 and 08951

B. Abelev et al. (ALICE Collaboration), Phys. Rev. C88 (2013) 044909.

[29] H. Högaasen, J. M. Richard and P. Sorba, Phys. Rev. D73 (2006) 054013.

[30] M. Abud (private comunication)

[31] F. Buccella, Phys. Rev. D98 (2018) 114011.

[32] F. Buccella and P. Sorba, Mod. Phys. Lett. A19 (2004) 1547.

[33] H. Högasen and P. Sorba, Nucl. Phys. B145 (1978) 119.

[34] L. Maiani, F. Piccinini, A. D. Polosa and V. Riquer, Phys. Rev. Lett. 93 (2004) 212002.

[35] G. 't Hooft, G. Isidori, L. Maiani, A. D. Polosa and V. Riquer, Phys. Lett. B662 (2008) 424.

[36] L. Maiani, F. Piccinini, A. D. Polosa and V. Riquer, Phys. Rev. D71 (2005) 014028. 